\title{
Topological canal foliations
}

\author{
By Gilbert Hector, Rémi Langevin and Paweł WalczaK
}

(Received May 30, 2017)

\begin{abstract}
Regular canal surfaces of $\mathbb{R}^{3}$ or $\mathbb{S}^{3}$ admit foliations by circles: the characteristic circles of the envelope. In order to build a foliation of $\mathbb{S}^{3}$ with leaves being canal surfaces, one has to relax the condition "canal" a little ("weak canal condition") in order to accept isolated umbilics. Here, we define a topological condition which generalizes this "weak canal" condition imposed on leaves, and classify the foliations of compact orientable 3-manifolds we can obtain this way.
\end{abstract}

\section{Introduction.}

In the literature, one can find several attempts to define, construct and possibly classify foliations on compact Riemannian manifolds with constant sectional curvature whose leaves enjoy some conformal property. There are mainly results of non existence, for example there do not exist

i) totally geodesic foliations of any codimension on negatively curved manifolds [Ze1], $[\mathrm{Ze} 2]$,

ii) codimension-one totally umbilical foliations on $\mathbb{S}^{n}$ (by purely topological reasons) or on negatively curved closed Riemannian manifolds [LW1],

iii) Dupin foliations on $\mathbb{S}^{3}$ or on compact hyperbolic 3-manifolds [LW1] whose leaves are pieces of Dupin cyclides (see below).

Recall that among all the local conformal invariants considered in the conformal geometry of surfaces (see, for example, [CSW]), one has conformal principal curvatures $\theta_{1}$, $\theta_{2}$ defined as the derivatives of the usual principal curvatures $k_{1}, k_{2}$ along corresponding suitably parameterized characteristic lines of curvature. A surface is Dupin if both of its conformal principal curvatures vanish and it is canal if this condition holds for one of them. This means that Dupin surfaces are canal in two ways: they carry two orthogonal families of characteristic lines. Canal surfaces are envelopes of one parameter families of spheres in the 3-dimensional space form; they carry characteristic circles, the intersections of the spheres defining them with the spheres of the derived family. These surfaces are important for computer aided geometric design (see $[\mathbf{K r}],[\mathbf{P P}]$, etc.). In order to enlarge the family of surfaces under consideration, one may allow isolated umbilical points; they will then be called geometric canal surfaces.

2010 Mathematics Subject Classification. Primary 57R30; Secondary 53C12.

Key Words and Phrases. foliation, canal surface, griddling.

The second and third authors were supported by the Polish NSC grant $\mathrm{N}^{\circ}$ 6065/B/H03/2011/40. 
Now a geometric canal foliation is any codimension-one foliation of a 3-dimensional manifold of constant sectional curvature such that all its leaves become - when lifted to the universal cover of the manifold under consideration-pieces of geometric canal surfaces. Certainly, $\mathbb{R}^{3}$ and flat 3 -tori admit canal foliations obtained from families of parallel affine planes. In [LW2], it is shown that the standard Reeb foliation of $\mathbb{S}^{3}$ is a geometric canal one and, conversely, that all canal foliations of $\mathbb{S}^{3}$ are not so far from Reeb foliations. More precisely, one has the following.

Theorem. Any foliation $\mathcal{F}$ of $\mathbb{S}^{3}$ by geometric canal surfaces is either a Reeb foliation with a unique toral leaf which is a Dupin cyclide or is obtained from such a Reeb foliation by inserting a zone $P \simeq \mathbb{T}^{2} \times[0,1]$ foliated by toral and cylindrical leaves.

Now our aim in the present paper is to enlarge the family of geometric canal surfaces or foliations by considering a purely topological condition: the existence of a griddled structure or griddling i.e. an orientable foliation or sub-foliation by circles possibly with isolated singularities. These griddled structures may be

- globally continuous and we will call the corresponding foliations griddled foliated manifolds,

- or piecewise continuous on compact saturated submanifolds with sudden discontinuities along finitely many compact leaves which will be analogues of Dupin cyclides defining our family of topological canal foliations which will include the previous family of geometric canal foliations of $\mathbb{S}^{3}$.

In Section 3 we construct a large class of griddled foliated manifolds and in Section 4, we show that this family is complete. For the sake of simplicity, we restrict to $C^{1}$ foliations and classify them up to $C^{0}$-isotopy or $C^{0}$-conjugation. Precisely we show the following:

Theorem A (see Theorem 4.14). A compact 3-manifold $M$ supports a griddled foliation of class $C^{1}$ if and only if $M$ is diffeomorphic to one of the following manifolds:

i) $\mathbb{D}^{2} \times[0,1], \mathbb{D}^{2} \times \mathbb{S}^{1}, \mathbb{S}^{2} \times[0,1]$ or $\mathbb{T}^{2} \times[0,1]$ if $\partial M \neq \emptyset$,

ii) $\mathbb{S}^{2} \times \mathbb{S}^{1}$ or any $\mathbb{S}^{1}$-bundle over $\mathbb{T}^{2}$ if $\partial M=\emptyset$.

Moreover if $M$ is either $\mathbb{D}^{2} \times[0,1]$ or $\mathbb{S}^{2} \times[0,1]$, all griddled structures on $M$ are $C^{0}$ isotopic while in the remaining cases, $M$ supports countably many different $C^{0}$-isotopy and $C^{0}$-conjugacy classes of griddled structures.

We introduce "topological canal" foliations in Section 5; these foliations may be smooth of any differentiability class and we classify the corresponding manifolds:

Theorem B (see Theorem 5.4). A topological canal foliation of class $C^{1}$ on a compact manifold is either griddled or obtained by gluing two or three compact griddled foliated pieces. Therefore a compact 3-manifold $M$ supports such a foliation if and only if $M$ is diffeomorphic to one of the following manifolds:

i) $\mathbb{D}^{2} \times[0,1], \mathbb{D}^{2} \times \mathbb{S}^{1}, \mathbb{S}^{2} \times[0,1]$ or $\mathbb{T}^{2} \times[0,1]$ if $\partial M \neq \emptyset$, 
ii) $\mathbb{S}^{2} \times \mathbb{S}^{1}, \mathbb{S}^{3}$ or any lens space, $\mathbb{T}^{3}$ or any $\mathbb{S}^{1}$-bundle over $\mathbb{T}^{2}$ if $\partial M=\emptyset$.

Moreover all manifolds $M$ listed in ii) support countably many different $C^{0}$-isotopy and $C^{0}$-conjugacy classes of topological canal foliations.

Finally we characterize geometric canal foliations among all topological canal foliations of $\mathbb{S}^{3}$ by observing that the characteristic circles of a geometric canal foliation on $\mathbb{S}^{3}$ define a "coherent system of meridians and parallels" on all toral leaves. We formalize this observation with the notion of "canal foliations of strong type" (see Definition 5.6) and using it we get

TheOREM C (see Theorem 5.8). A topological canal foliation $\mathcal{F}$ of class $C^{2}$ on $\mathbb{S}^{3}$ is $C^{0}$-conjugate to a geometric one if and only if it is of strong type.

As an immediate consequence of Theorem B, we also observe that no compact hyperbolic manifold can support topological canal foliations, corroborating the nonexistence of Dupin foliations on such manifolds already established in [LW1].

\section{Griddled surfaces and foliations.}

Manifolds considered in this paper will be of dimension 2 or 3, connected, smooth and orientable. For the sake of simplicity we will assume that all foliations are of class $C^{1}$ so that we can use the fundamental result of Epstein concerning finiteness of holonomy groups for compact codimension 2 foliations on 3-manifolds (see [E] and [EMS]). Moreover we suppose that all foliations are orientable and transversely orientable. If a manifold $M$ of dimension 2 or 3 has non empty boundary and $N$ is a connected component of $\partial M$, we will also assume that

i) any codimension 1 foliation $\mathcal{F}$ on $M$ is either tangent or transverse to $N$; accordingly we say that $N$ is a tangential or a transversal component of $\partial M$,

ii) any codimension 2 foliation $\mathcal{C}$ is always tangent to any boundary component $N$.

Note that $\partial M$ may have simultaneously tangential and transversal components. Moreover, in order to facilitate our constructions, we will exceptionally consider also the product $\mathbb{D}^{2} \times[0,1]$ with the canonical foliation by closed disks; in this case, the boundary of $\mathbb{D}^{2} \times[0,1]$ splits into a tangential part $\mathbb{D}^{2} \times\{0,1\}$ and a transversal part $\partial \mathbb{D}^{2} \times[0,1]$.

We believe that our results can be adapted to $C^{0}$ foliations but in this context, proofs would become long and tedious: therefore we prefer to restrict ourselves to the $C^{1}$-case.

Definition 2.1. A griddled structure on a surface $L$ is a 1-dimensional foliation $\mathcal{C}$ (with singularities) such that

i) any singularity of $\mathcal{C}$ is isolated,

ii) any regular leaf of $\mathcal{C}$ is homeomorphic to $\mathbb{S}^{1}$. 
Similarly, a griddled structure on a foliated 3-manifold $(M, \mathcal{F})$ will be an orientable subfoliation $\mathcal{C}$ of the codimension 1 foliation $\mathcal{F}$ which induces by restriction a griddled structure on each leaf $L$ of $\mathcal{F}$.

We will say that $L$ and $(M, \mathcal{F})$ are griddled.

This notion is inspired by the geometry of regular canal surfaces, possibly with isolated umbilical points, which are griddled by their characteristic circles.

EXAmple 2.2. Canonical griddled structures on surfaces.

Recall that an action of $\mathbb{S}^{1}$ is called semi-free if any non trivial isotropy subgroup is the whole of $\mathbb{S}^{1}$. Now a semi-free action of $\mathbb{S}^{1}$ on a surface defines a griddled structure provided that its singularities are isolated; we call such a structure canonical. Examples of canonical griddled structures on surfaces (see Figure 1) are defined by the following semi-free actions:

(1) the action of the group of rotations around the origin of the plane $\mathbb{R}^{2}$ or the unit disk $\mathbb{D}^{2}$; the action of the group of rotations of the unit sphere $\mathbb{S}^{2}$ around the vertical axis $\overrightarrow{O z}$ of $\mathbb{R}^{3}$; these structures have either one or two singularities,

(2) the natural free action of the first factor on the annulus $\mathbb{A}=\mathbb{S}^{1} \times[0,1]$, the cylinders $\mathbb{S}^{1} \times \mathbb{R}$ or $\left.\left.\mathbb{S}^{1} \times\right] 0,1\right]$, the torus $\mathbb{T}^{2}=\mathbb{S}^{1} \times \mathbb{S}^{1} ;$ all four structures are regular, without singularities.
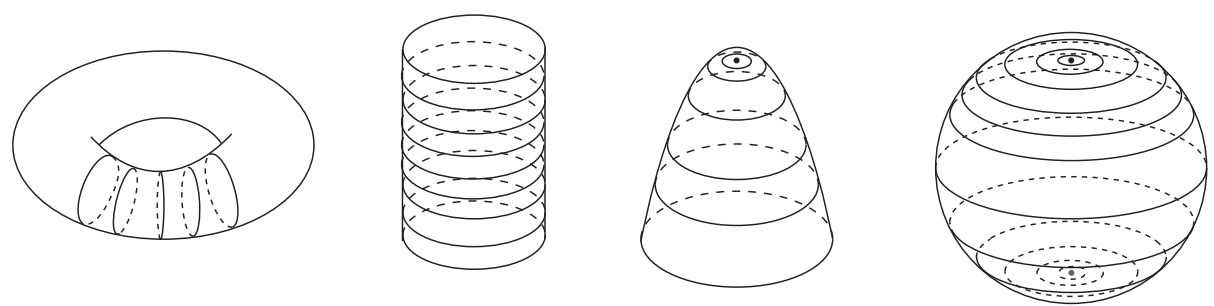

Figure 1. Canonical griddled structures.

Next we show that any griddled surface is topologically conjugate to one of these canonical surfaces.

LEMMA 2.3. Any singularity of a connected griddled surface $(L, \mathcal{C})$ is a center and $(L, \mathcal{C})$ is topologically conjugate to one of the previous canonical griddled surfaces.

Proof. As singularities are isolated, the set $\Sigma$ of singularities of $\mathcal{C}$ is a countable discrete subset of $L$ and $\hat{L}=L \backslash \Sigma$ is a connected surface foliated by circles. Due to the transverse orientability, the restriction $\hat{\mathcal{C}}$ of $\mathcal{C}$ to $\hat{L}$ is without holonomy thus a locally trivial circle bundle so that $\hat{L}$ is homeomorphic to $\left.\left.\mathbb{S}^{1} \times\right] 0,1\right], \mathbb{S}^{1} \times \mathbb{R}$ or $\mathbb{T}^{2}$. Moreover, $\hat{L}$ is an open dense subset of $L$ and any singularity of $\mathcal{C}$ can be seen as an end of $\hat{L}$. We conclude that, either $L$ is the torus $\mathbb{T}^{2}$ or $L$ has genus 0 and therefore is a cylinder, a plane or a disk, or it is a 2 -sphere with respectively 0,1 or 2 singularities. 
Of course, the induced structure $\hat{\mathcal{C}}$ is conjugate to the canonical regular structure of $\hat{L}$ and because $\Sigma$ is identified with the set of ends of $\hat{L}$, any conjugacy extends to $\Sigma$. It follows that any griddled structure is conjugate to a canonical one; in particular any singularity is a center.

The following observation will be helpful for the classification of griddled 3-manifolds:

REMARK 2.4. If a griddled surface $(L, \mathcal{C})$ admits a loop $\gamma$ contained in the regular part $\hat{L}$ and transverse to $\hat{\mathcal{C}}$, then $(L, \mathcal{C})$ is a canonically griddled torus and $\gamma$ is not null homotopic in $L$.

REMARK 2.5. Further, we notice that the group of orientation preserving diffeotopies of the griddled surface $(L, \mathcal{C})$ reduces to

i) the identity if $L \neq \mathbb{T}^{2}$,

ii) the cyclic group generated by the Dehn twist along the typical fiber of $\mathcal{C}$ if $L=\mathbb{T}^{2}$.

\section{Griddled foliated 3-manifolds: examples.}

Here, we introduce a large class of compact griddled foliated 3-manifolds $(M, \mathcal{F}, \mathcal{C})$. According to Lemma 2.3, any leaf of $\mathcal{F}$ is one of the griddled surfaces listed in Example 2.2 and we denote by $\Sigma$ the singular set of $\mathcal{C}$.

First, we describe three special families of griddled 3-manifolds.

EXAMPLE 3.1. Griddled fibrations.

Let $\left(L, \mathcal{C}_{0}\right)$ be one of the compact griddled surfaces described in Example 2.2 and consider the trivial fibration $\mathcal{F}$ defined by $\pi: M=L \times J \rightarrow J$ where $J$ is either $[0,1]$ or $\mathbb{S}^{1}$ when $L$ is $\mathbb{D}^{2}, \mathbb{S}^{2}$ or $\mathbb{T}^{2}$ but $J=\mathbb{S}^{1}$ when $L=\mathbb{A}$. The canonical semi-free action of $\mathbb{S}^{1}$ on $L$ lifts to the fibers of $\pi$ defining a semi-free action of $\mathbb{S}^{1}$ on $M$ : it generates a subfoliation $\mathcal{C}$ of $\mathcal{F}$ which is a griddled structure called the canonical griddled structure of $(M, \mathcal{F})$.

So far we have obtained seven griddled 3-manifolds: the corresponding singular set is homeomorphic respectively to $J$, the sum of two copies of $J$ or is empty.

The second family is that of Reeb components. They will play an important role in the sequel, therefore we start recalling some well known properties of these foliations.

EXAmPLE 3.2. Griddled Reeb components.

(1) Let $(x, y)$ be the usual coordinate system of $[-1,+1] \times \mathbb{R}$, and let $\Omega_{ \pm}$be the two non singular one forms on the annulus $\mathbb{A}=[-1,1] \times \mathbb{S}^{1}$ which lift to $[-1,1] \times \mathbb{R}$ as

$$
\Omega_{ \pm}=a(x) d y \pm x d x
$$

where $a(x)$ is a smooth positive function on $[-1,+1]$ verifying $a(-x)=a(x)$ for any $x$ and $a(x)=0$ exactly for $x= \pm 1$. For a fixed function $a(x)$, these forms define foliations which are tangent to the boundary, symmetric with respect to the transversal circle $\Sigma=\{0\} \times \mathbb{S}^{1}$ and such that any interior leaf is diffeomorphic to $\mathbb{R}$ and spirals 
towards the two boundary circles. They are not isotopic but differentiably conjugate by the diffeomorphism $\psi$ of $\mathbb{A}$ induced by $\tilde{\psi}:[-1,+1] \times \mathbb{R} \rightarrow[-1,+1] \times \mathbb{R}$ given by $\tilde{\psi}(x, y)=(x,-y)$.

Any such foliation is called a 2-dimensional Reeb component (also called Poincaré component in [LW2], see Figure 2), positive or negative depending on the choice of the $\operatorname{sign} \pm$.
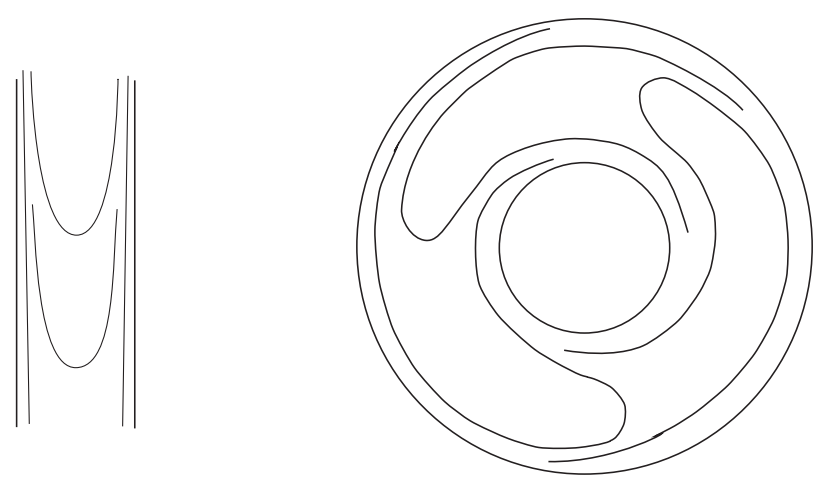

Figure 2. Reeb component.

(2) Now note that the holonomy transformation associated to the boundary circles of such a Reeb component depends strongly on the choice of the function $a(x)$; for example it will be flat, that is differentiably tangent to the identity, if $a(x)=e^{-1 /\left(1-x^{2}\right)}$ but have non trivial first derivative if $a(x)=1-x^{2}$. As this derivative is invariant by $C^{1}$-conjugacy, it follows that there are infinitely many $C^{1}$-conjugacy classes of these Reeb components but one verifies that they are all $C^{0}$-conjugate (see $[\mathbf{H H}]$ ). Note also that there are two $C^{0}$-isotopy classes of these Reeb components characterized by the sign \pm in the definition of $\Omega_{ \pm}$.

(3) Next, rotating a 2-dimensional Reeb component $\left(\mathbb{A}, \Omega_{ \pm}\right)$around the circle $\Sigma$, one generates the solid torus $\Theta$ endowed with a 2-dimensional foliation $\mathcal{R}_{ \pm}$which is griddled by construction with $\Sigma$ as set of singularities; the boundary torus is a compact leaf of $\mathcal{R}_{ \pm}$all interior leaves being proper planes spiraling onto the boundary. It will be called a griddled Reeb component (see Figure 3).

By construction, isotopy and conjugacy in the family of griddled Reeb components reduce to the corresponding relation in the family of Reeb Components of dimension 2 . Thus as an immediate consequence of (2) above, there are infinitely many $C^{1}$-conjugacy classes but only two $C^{0}$-isotopy classes and one $C^{0}$-conjugacy class of griddled Reeb components.

(4) Finally observe that the boundary torus $\partial \Theta$ of a griddled Reeb component $\Theta$ is a canonically griddled torus. Thus identifying the boundaries of two griddled Reeb components, we define a griddled structure on $\mathbb{S}^{2} \times \mathbb{S}^{1}$. The identification is defined up to a Dehn twist of $\partial \Theta$ along the fiber of the griddling of $\partial \Theta$, this twist extends as a griddled isomorphism of $\Theta$ and consequently the gluing of two Reeb components will be independent of the gluing map. The resulting structure will be called a canonical griddled Reeb structure of $\mathbb{S}^{2} \times \mathbb{S}^{1}$. The underlying codimension 1 foliation $\mathcal{F}$ is the result of gluing 

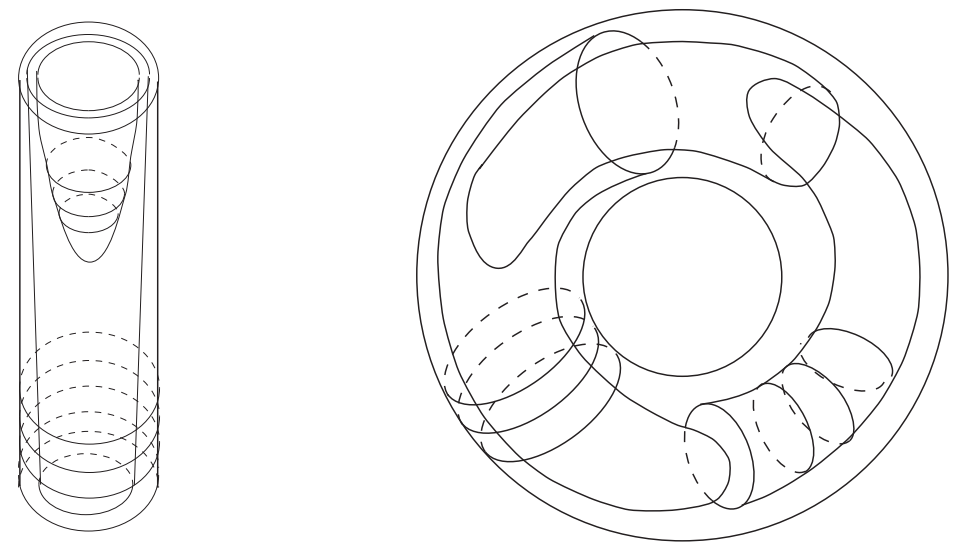

Figure 3. Griddled Reeb component.

two Reeb components and it is not difficult to see that there will be two $C^{0}$-conjugacy classes of such Reeb structures depending on the fact that we glue two Reeb components of the same sign or not.

EXAmple 3.3. Regular griddled structures.

(1) Consider an orientable $\mathbb{S}^{1}$-bundle: $\mathbb{S}^{1} \longrightarrow M \stackrel{\pi}{\longrightarrow} B$ over a compact connected surface $B$ endowed with a 1-dimensional foliation $\mathcal{L}$ without singularities. The Euler characteristic of $B$ vanishes so $B$ is either the 2-torus or the annulus $\mathbb{A}=\mathbb{S}^{1} \times[0,1]$ and $\mathcal{F}=\pi^{*}(\mathcal{L})$ is a codimension 1 foliation on $M$ which is griddled by the $\mathbb{S}^{1}$-fibration: its leaves are cylinders or tori and its singular set is empty. This family includes the canonical structures of $\mathbb{T}^{2} \times[0,1]$ or $\mathbb{T}^{2} \times \mathbb{S}^{1}$; we call it the family of canonical regular griddled structures; all these examples are defined by a free action of $\mathbb{S}^{1}$.

In particular, if $B=\mathbb{A}$, the $\mathbb{S}^{1}$-bundle is trivial, $M=\mathbb{T}^{2} \times[0,1]$ and the foliation $\mathcal{F}$ is any foliation by cylinders and tori; due to our conventions in Section $2, \mathcal{L}$ and $\mathcal{F}$ may be tangent to one boundary component and transverse to the other or tangent [resp. transverse] to both.

(2) It is interesting to observe that the isotopy [or conjugacy] class of a canonical regular griddled structure $(M, \mathcal{F}, \mathcal{C})$ is determined by the isotopy [or conjugacy] class of the corresponding 1-dimensional foliation $(B, \mathcal{L})$. We refer to $[\mathbf{H C}]$ for a classification of these foliations on $\mathbb{T}^{2}$ or $\mathbb{A}$. Indeed they are defined essentially by the "algebraic number" of 2-dimensional Reeb components (compare Example 3.2 above). In particular, there are infinitely many $C^{0}$-conjugacy classes of such foliations and thus also of griddled structures on the corresponding $\mathbb{S}^{1}$-bundles (see also $[\mathbf{K n} \mathbf{1}]$ ).

(3) There are two special cases when the foliation $\mathcal{L}$ on the annulus $\mathbb{A}$ is either a 2-dimensional Reeb component or a so-called "spiraling zone" defined by the suspension of a diffeomorphism $g:[0,1] \rightarrow[0,1]$ with $\{0,1\}$ as set of fix points. In each case there is only one $C^{0}$-conjugacy class and thus exactly one $C^{0}$-conjugacy class for the corresponding regular griddled structure on $\mathbb{A} \times \mathbb{S}^{1}$. 
(4) Finally note that if $(B, \mathcal{L})$ is a Denjoy foliation (with so called exceptional leaves), then any $\mathbb{S}^{1}$-bundle over $B$ will produce a "Denjoy type" griddled structure: the codimension 1 leaves will be cylinders and some of them are neither proper nor dense (see $[\mathbf{H H}]$ for more details).

Next we generalize the gluing procedure used for the construction of the griddled Reeb structures on $\mathbb{S}^{2} \times \mathbb{S}^{1}$ :

Construction 3.4. Observe that for all the compact griddled manifolds with boundary described in Examples 3.1, 3.2 or 3.3, any boundary component is a canonically griddled disk, cylinder, sphere or torus and we will obtain more examples by sticking together such basic pieces by means of $C^{1}$-diffeomorphisms of the boundary pieces compatible with the codimension 1 foliations and preserving the griddled structures. Moreover, in order to obtain a global $C^{1}$-structure, it will be necessary, when gluing two tangential boundary components, to assume that the derivatives of the corresponding holonomy transformations coincide.

Finally note that there are two types of gluings: either we take two basic pieces with isomorphic boundary components and glue them along these two components or we take a connected manifold whose boundary has two isomorphic connected components and identify them.

Next we describe some particularly interesting examples:

EXAMPLE 3.5. Griddled manifolds obtained by gluing basic pieces.

(1) First, suppose that $M$ is one of the four manifolds $\mathbb{D}^{2} \times[0,1], \mathbb{S}^{2} \times[0,1], \mathbb{T}^{2} \times[0,1]$ or $\mathbb{D}^{2} \times \mathbb{S}^{1}$ endowed with its canonical griddled structure coming from the canonical griddled structure of $\mathbb{D}^{2}, \mathbb{S}^{2}$ or $\mathbb{T}^{2}$ (see Examples 3.1 and 2.2).

a) If we glue two copies of $M$ along tangential boundary disks, spheres or tori, we obtain the same manifold $M$ with a canonical structure; in particular the result does not depend on the gluing map.

b) We may also identify the transversal boundary components $\partial \mathbb{D}^{2} \times[0,1]$ of two copies of $\mathbb{D}^{2} \times[0,1]$ producing a canonical $\mathbb{S}^{2} \times[0,1]$. Similarly, we obtain a canonical $\mathbb{S}^{2} \times \mathbb{S}^{1}$ when sticking together two copies of the canonical $\mathbb{D}^{2} \times \mathbb{S}^{1}$.

c) Finally we identify the two tangential boundary components of one copy of $M$. For $M=\mathbb{D}^{2} \times[0,1]$, we get a canonical $\mathbb{D}^{2} \times \mathbb{S}^{1}$; the situation is similar for $M=\mathbb{S}^{2} \times[0,1]$ due to the first observation in 2.5 and we get a canonical $\mathbb{S}^{2} \times \mathbb{S}^{1}$. Last for $M=\mathbb{T}^{2} \times[0,1]$, the gluing is homotopic to a multiple of the Dehn twist (see Remark 2.5) and the resulting griddled manifold $(\tilde{M}, \mathcal{F}, \mathcal{C})$ is any $\mathbb{S}^{1}$-bundle over $\mathbb{T}^{2}$ which is also a $\mathbb{T}^{2}$-fibration over $\mathbb{S}^{1}$.

(2) A little bit more generally, we can stick together two copies of $\mathbb{T}^{2} \times[0,1]$ endowed with a priori different regular structures provided they admit isomorphic boundary components (tangential or transversal); we obtain a new regular structure. The result is similar when identifying the two boundary components of some griddled $\mathbb{T}^{2} \times[0,1]$ assuming they are griddled isomorphic. 
(3) So far, our previous descriptions in (1) and (2), did not produce any new example. Fortunately, the situation will be different when using Reeb components as basic pieces. To generalize the construction of the Reeb structure on $\mathbb{S}^{2} \times \mathbb{S}^{1}$, we consider a regular griddled manifold $\left(\mathbb{T}^{2} \times[0,1], \mathcal{F}, \mathcal{C}\right)$ with $\mathcal{F}$ tangent to the boundary and stick it together with one or two griddled Reeb components. The global manifold $M$ we obtain, will be either $\mathbb{D}^{2} \times \mathbb{S}^{1}$ or $\mathbb{S}^{2} \times \mathbb{S}^{1}$ but due to the presence of the factor $\mathbb{T}^{2} \times[0,1]$, this construction provides countably many different isotopy or conjugacy classes of griddled structures on $M$.

Of course in order to obtain $C^{1}$-examples, it will be necessary to control the holonomy groups of the tangential boundary pieces.

\section{Griddled foliated 3-manifolds: classification.}

Here, we want to show that the list of compact griddled foliated manifolds described in the previous Section 3 is complete; that is any griddled foliated manifold is conjugate to one of them. To do so we first describe some elementary properties of these manifolds.

So let $(M, \mathcal{F}, \mathcal{C})$ be a compact griddled 3-manifold. Its singular set $\Sigma$ is closed thus $\hat{M}=M \backslash \Sigma$ is an open subset of $M$ with a regular foliation $\hat{\mathcal{C}}$ by circles. For $x \in \hat{M}$ we denote by $L_{x}$ the leaf of $\mathcal{F}$ through $x$ and by $c_{x}$ the corresponding leaf of $\mathcal{C}$. We first study the holonomy groups $\operatorname{hol}\left(c_{x}, \mathcal{C}\right)$ and $\operatorname{hol}\left(L_{x}, \mathcal{F}\right)$ of $c_{x}$ and $L_{x}$ with respect to $\mathcal{C}$ and $\mathcal{F}$ respectively:

Lemma 4.1. For any $x \in \hat{M}$, there is an injective group homomorphism

$$
\varphi: \operatorname{hol}\left(c_{x}, \mathcal{C}\right) \rightarrow \operatorname{hol}\left(L_{x}, \mathcal{F}\right)
$$

In particular if $L_{x}$ is a plane or a sphere, the group $\operatorname{hol}\left(c_{x}, \mathcal{C}\right)$ is trivial.

Proof. (1) Fix $x \in \hat{M}$, there exists an open cube $U \subset \hat{M}$ which is a neighborhood of $x$ and which is distinguished for both foliations $\mathcal{F}$ and $\mathcal{C}$. Moreover there exists a system of coordinates $(u, v, t)$ on $U$ such that the restrictions

a) $\mathcal{F}_{U}$ is defined by the equation $d t=0$,

b) $\mathcal{C}_{U}$ is defined by $d u=d t=0$.

Finally take a global section $T_{x}$ of $\mathcal{C}_{U}$ through $x$. Then as $\mathcal{C}$ is a subfoliation of $\mathcal{F}$, the holonomy transformation associated to the loop $c_{x}$ with respect to $\mathcal{C}$ is a local transformation $h_{x}:\left(T_{x}, x\right) \rightarrow\left(T_{x}, x\right)$ which preserves the foliation induced on $T_{x}$ by $\mathcal{F}$. This means that in our system of coordinates $h_{x}$ can be written as

$$
h_{x}(y)=h_{x}(u, t)=[f(u, t), g(t)]
$$

for any point $y=(u, t)$ in $T_{x}$ sufficiently close to $x=(0,0)$, where $f$ and $g$ are two real valued functions. The germ $[g]$ of $g$ defines an element in $\operatorname{hol}\left(L_{x}, \mathcal{F}\right)$ and we define $\varphi$ by setting $\varphi\left(\left[h_{x}\right]\right)=[g]$, where $\left[h_{x}\right]$ is the germ of $h_{x}$ in $x$; it is of course a group homomorphism. 
(2) To prove the injectivity of $\varphi$, observe that $\left[h_{x}\right]$ belongs to the kernel of $\varphi$ means that the germ $[g]$ is trivial that is $g(t)=t$ in a neighborhood of 0 and for any $y=(u, t)$,

$$
h_{x}(y)=h_{x}(u, t)=[f(u, t), t] .
$$

Moreover, for $t=0$, the function $f(u, 0)$ defines the holonomy transformation associated to the loop $c_{x}$ with respect to the foliation $\mathcal{C}_{L_{x}}$ induced by $\mathcal{C}$ on the leaf $L_{x} \in \mathcal{F}$. This latter foliation is a transversely oriented foliation by circles on a surface thus without holonomy, $\operatorname{hol}\left(c_{x}, \mathcal{C}_{L_{x}}\right)$ is trivial and

$$
f(u, 0)=u
$$

in a neighborhood of $x$ in $L_{x}$.

As the similar observation holds for any $y=(u, t)$ close to $x$ and the foliation $\mathcal{C}_{L_{y}}$ induced by $\mathcal{C}$ on the leaf $L_{y} \in \mathcal{F}$, we get

$$
h_{x}(y)=h_{x}(u, t)=[f(u, t), t]=(u, t)=y
$$

and $\operatorname{hol}\left(c_{x}, \mathcal{C}\right)$ is trivial. The rest follows.

Lemma 4.2. If $\Sigma=\emptyset$, the griddled structure $\mathcal{C}$ is a locally trivial $\mathbb{S}^{1}$-bundle: $\mathbb{S}^{1} \longrightarrow M \stackrel{\pi}{\longrightarrow} B$ defining a canonical regular structure as in Example 3.3.

In particular, for $J$ equal to $[0,1]$ or $\mathbb{S}^{1}$, any griddled structure supported by the trivial fibration $\pi: \mathbb{T}^{2} \times J \rightarrow J$ is a canonical one.

Proof. As $M$ is compact (possibly with boundary) and $\mathcal{C}$ is of class $C^{1}$, the fundamental theorem of $[\mathbf{E}]$ implies that $\mathcal{C}$ may be defined by a locally free action of $\mathbb{S}^{1}$ so that any holonomy group $\operatorname{hol}\left(c_{x}, \mathcal{C}\right)$ is finite cyclic. On the other hand, as $\mathcal{F}$ is transversely orientable, any non trivial element of $\operatorname{hol}\left(L_{x}, \mathcal{F}\right)$ has infinite order so that the image of the homomorphism $\varphi$ provided by Lemma 4.1 is trivial implying that $\operatorname{hol}\left(c_{x}, \mathcal{C}\right)$ is trivial. This means that the action of $\mathbb{S}^{1}$ is free and $\mathcal{C}$ is a locally trivial fibration. We conclude that $\mathcal{C}$ is canonical.

In the particular case when $M=\mathbb{T}^{2} \times J$, any bundle $\mathbb{S}^{1} \rightarrow M \rightarrow B$ over a surface $B$ is determined by the common homotopy class of the fibers: it is indeed a trivial bundle therefore conjugate to a canonical regular structure. Our proof is complete.

REMARK 4.3. The previous result applies in particular to the case of $\mathbb{T}^{2} \times[0,1] \cong$ $\mathbb{A} \times \mathbb{S}^{1}$ where $\mathbb{A}$ is foliated as a 2-dimensional Reeb component or a spiraling zone (compare with point (3) in Example 3.3).

The proof of the previous lemma uses strongly the fact that $M$ is compact; in particular the argument does not extend a priori to $(\hat{M}, \hat{\mathcal{C}})$. Nevertheless we will see later that, in any case, all regular leaves of $\mathcal{C}$ have trivial holonomy. Before proving this point we focus on the description of the singular set $\Sigma$.

LEMMA 4.4. If $\mathcal{F}$ is the trivial fibration by disks $\pi: \mathbb{D}^{2} \times J \rightarrow J$, where $J$ is either $[0,1]$ or $\mathbb{S}^{1}$, then $\mathcal{C}$ is topologically conjugate to the canonical griddled structure on $\mathbb{D}^{2} \times J$. 
In particular $\Sigma$ is a topological submanifold (possibly not differentiable) of $\mathbb{D}^{2} \times J$.

Proof. By definition, any fiber of $\pi$ is endowed with a structure conjugate to the canonical griddled structure of $\mathbb{D}^{2}$. Therefore, there is a section $\sigma$ of $\pi$ such that $\Sigma=\sigma(J)$ and due to the continuity of $\mathcal{C}$, this section is continuous and $\Sigma$ is a continuous arc transverse to $\mathcal{F}$; we may identify it with $\{0\} \times J$ by means of a fiber preserving isotopy.

On the other hand, it is not difficult to see that there exists a family of fiberwise conjugacies which depend continuously on the transverse parameter defined by $J$ thus defining a global conjugacy. Indeed according to Lemma 4.1, the foliation $\hat{\mathcal{C}}$ has trivial holonomy, therefore the length (for some Riemannian metric) of its leaves defines a $C^{1}$ function $\alpha$ that extends continuously to $\{0\} \times J$. Then take a unit vector field $X$ tangent to $\hat{\mathcal{C}}$ and note that $\alpha X$ is a periodic vector field whose flow defines an $\mathbb{S}^{1}$ action generating $\hat{\mathcal{C}}$. By averaging, one gets a $C^{1}$-Riemannian metric on $\hat{M}$ invariant by this action; one can see this metric as a $C^{1}$ path of invariant Riemannian metrics on the punctured disk and the wanted conjugacy is then given by a parametric version of the Gauss Lemma expressing a Riemannian metric in polar coordinates.

LEMma 4.5. Any connected component $\Sigma_{0}$ of $\Sigma$ is homeomorphic to $[0,1]$ or $\mathbb{S}^{1}$ and admits a closed tubular neighborhood $\tau\left(\Sigma_{0}\right)$ which is $\mathcal{C}$-saturated and isomorphic to a canonically griddled $\mathbb{D}^{2} \times[0,1]$ or $\mathbb{D}^{2} \times \mathbb{S}^{1}$.

Globally, $\Sigma$ is a compact 1-manifold transverse to $\mathcal{F}$; if $M$ is closed, it is a transverse link.

Proof. Let $U_{0}$ be a $\mathcal{F}$-distinguished open cube which is a neighborhood of a point $z_{0} \in \Sigma_{0}$ and let $P_{0} \subset U_{0}$ be the $\mathcal{F}$-plaque of $U_{0}$ containing $z_{0}$. According to Lemma 2.3, we know that $z_{0}$ is a center for the local restriction of $\mathcal{C}$ to $P_{0}$ and that $L_{z_{0}}$ is a plane or a sphere. Then there exists a point $x \in P_{0}$ such that $c_{x}$ bounds a closed disk $D_{x} \subset P_{0}$ with $z_{0} \in D_{x}$.

Assume that $z_{0}$ belongs to the interior of $M$; again by Lemma 4.1, the loop $c_{x}$ has trivial $\mathcal{C}$-holonomy. This means that any arc $\gamma:[-1,1] \rightarrow T_{x} \subset U_{0}, \gamma(0)=x$, transverse to $\mathcal{F}$ will be pointwise fixed by the holonomy transformation $h_{x}$ thus generates a compact cylinder $\Gamma=\cup_{t \in[-1,+1]} c_{\gamma(t)}$ transverse to $\mathcal{F}$. Each circle $c_{\gamma(t)}$ bounds a $\mathcal{C}$-saturated disk $D_{\gamma(t)}$ contained in $P_{\gamma(t)}$ and by Lemma 2.3, $D_{\gamma(t)}$ supports a canonical griddled structure with a unique singularity $z_{\gamma(t)}$. Consequently according to Lemma $4.4, \Theta=\cup_{t \in[-1,1]} D_{\gamma(t)}$ is isomorphic to the canonically griddled $\mathbb{D}^{2} \times[0,1]$ (see Figure 4 ). We proceed in a similar way when $z_{0}$ belongs to $\partial M$ with the difference that $\gamma$ will be parametrized by $[0,1]$ instead of $[-1,+1]$. We conclude that $\Sigma$ is a 1 -dimensional manifold transverse to $\mathcal{F}$ and to the boundary when $\partial M \neq \emptyset$.

Finally, we globalize the previous argument as follows: we take a global tubular neighborhood $U$ of $\Sigma_{0}$ and a transverse arc $\gamma \subset U$ parametrized by $\Sigma_{0}$ which meets every fiber of $U$ in exactly one point. Again we may assume that the corresponding circles $c_{\gamma}(t)$ are contained in $U$ and dealing as before, we construct a $\mathcal{C}$-saturated tubular neighborhood of $\Sigma_{0}$ contained in $U$. It is the wanted neighborhood $\tau\left(\Sigma_{0}\right)$. 

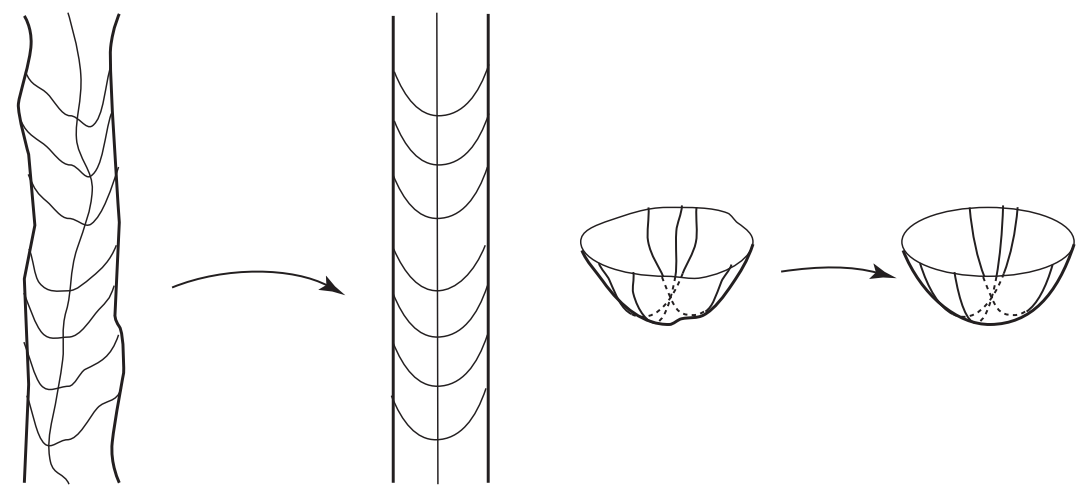

Figure 4. Conjugation of a neat tubular neighborhood of closed singular curve of the griddling with the standard one.

Definition 4.6. The tubular neighborhood $\tau\left(\Sigma_{0}\right)$ provided by Lemma 4.5 will be called a neat tubular neighborhood of $\Sigma_{0}$. If $\Sigma$ has several connected components, we define a neat tubular neighborhood $\tau(\Sigma)$ of $\Sigma$ as being the topological sum of pairwise disjoint neat neighborhoods of each component.

LEMMA 4.7. In any case, the restriction $\hat{\mathcal{C}}$ of $\mathcal{C}$ is a locally trivial fibration by circles of $\hat{M}=M \backslash \Sigma$.

Proof. Indeed for any point $x \in \hat{M}$, there exists a neat tubular neighborhood $\tau(\Sigma)$ of $\Sigma$ which does not intersect the circle $c_{x}$. Applying Lemma 4.2 to the compact griddled submanifold $M \backslash \operatorname{int}[\tau(\Sigma)]$, we see that $c_{x}$ has trivial holonomy. As this holds for any $x \in \hat{M}$, we conclude that $\hat{\mathcal{C}}$ has trivial holonomy thus is a fibration by circles.

There are two more particular cases of interest:

LEMma 4.8. If $\mathcal{F}$ is the trivial fibration by spheres $\pi: M=\mathbb{S}^{2} \times J \rightarrow J$, where $J$ is either $[0,1]$ or $\mathbb{S}^{1}$, then $\mathcal{C}$ is topologically conjugate to the canonical griddled structure on $\mathbb{S}^{2} \times J$.

In particular, the singular set $\Sigma$ has two connected components.

Proof. In any case, the restriction of $\pi$ to $\Sigma$ is a two fold covering of $J$. Now, there are two possibilities:

(1) First, if $J=[0,1]$, the singular set $\Sigma$ has two connected components $\Sigma_{0}$ and $\Sigma_{1}$ both homeomorphic to $[0,1]$ and if $\tau\left(\Sigma_{0}\right)$ is a neat tubular neighborhood of $\Sigma_{0}$, then $M \backslash \operatorname{int}\left[\tau\left(\Sigma_{0}\right)\right]$ is a neat neighborhood $\tau\left(\Sigma_{1}\right)$ of $\Sigma_{1}$. Gluing these neighborhoods together, we obtain a canonical griddled $\mathbb{S}^{2} \times[0,1]$.

(2) For $J=\mathbb{S}^{1}$, we claim that $\Sigma$ has still two connected components and the restriction of $\pi$ to any of these components is a homeomorphism onto $\mathbb{S}^{1}$. Indeed, assume that $\Sigma$ is connected; it still cuts each fiber of $\pi$ in two points. Take a neat tubular neighborhood $\tau(\Sigma)$, the restriction of $\pi$ to the compact manifold $N=M \backslash \operatorname{int}[\tau(\Sigma)]$ will define an orientable fibration of $N$ over the circle with fiber the annulus $\mathbb{A}$. But $\partial \tau(\Sigma)$ 
being connected, so will be $\partial N$ and thus the monodromy map $\phi: \mathbb{A} \rightarrow \mathbb{A}$ exchanges the two oriented boundary components of $\mathbb{A}$. Since these boundary components are circles belonging to $\mathcal{C}$, we get a contradiction with the assumption that $\mathcal{C}$ is orientable.

Now the argument already used in (1) above applies showing that the griddled structure on $\mathbb{S}^{2} \times \mathbb{S}^{1}$ is the canonical one.

LEMma 4.9. Any griddled structure $\mathcal{C}$ supported by a Reeb component is conjugate to a canonical one.

Proof. Take a griddled Reeb component $\left(\mathbb{D}^{2} \times \mathbb{S}^{1}, \mathcal{R}, \mathcal{C}\right)$. As each planar leaf of $\mathcal{R}$ contains exactly one singularity, it follows that the singular set $\Sigma$ of $\mathcal{C}$ is a closed transversal to $\mathcal{R}$ ambient isotopic with the transversal $\{0\} \times \mathbb{S}^{1}$ by an isotopy preserving each individual leaf of $\mathcal{R}$. Take a neat tubular neighborhood $\tau(\Sigma)$ of $\Sigma$, the compact submanifold

$$
N=\left(\mathbb{D}^{2} \times \mathbb{S}^{1}\right) \backslash \operatorname{int}[\tau(\Sigma)]
$$

is homeomorphic to $\mathbb{T}^{2} \times[0,1]$ with the foliation induced by $\mathcal{R}$ tangent to one boundary component and transverse to the other. The traces on $N$ of the planar leaves are cylinders homeomorphic to $\mathbb{S}^{1} \times[0,+\infty$ [ while the restriction of $\mathcal{C}$ is a circle bundle over $\mathbb{A}=$ $N / \mathcal{C}$. Except the border leaves, all leaves of the one-dimensional foliation $\mathcal{L}=\mathcal{R} / \mathcal{C}$ are homeomorphic to $[0,+\infty[$, transverse to one boundary component of $\mathbb{A}$ and spiraling around the other. We say that $\mathcal{L}$ is the "suspension" of a contraction $[0,1] \rightarrow\left[0, x_{0}\right], 0<$ $x_{0}<1$ (see Figure 5). We recover $\mathbb{D}^{2} \times \mathbb{S}^{1}$ by gluing together $\tau(\Sigma)$ and $N$ along their transverse boundary tori.

Now, take another griddled Reeb structure $\mathcal{C}_{*}$ supported by the same foliation $\mathcal{R}$ and consider the corresponding decomposition $\left[\tau\left(\Sigma_{*}\right), N_{*}\right]$ of the Reeb component $\left(\mathbb{D}^{2} \times \mathbb{S}^{1}, \mathcal{R}\right)$ obtained from $\mathcal{C}_{*}$. The 1-dimensional foliation $\mathcal{L}_{*}$ is conjugate to $\mathcal{L}$, because both are "suspension" of contractions as above. Therefore the two griddled manifolds $N$ and $N_{*}$ are conjugate as well. The two neighborhoods $\tau(\Sigma)$ and $\tau\left(\Sigma_{*}\right)$ are conjugate according to Lemma 4.5. Finally, these conjugacies are compatible with the gluing maps and provide a global conjugacy between the two structures $\mathcal{C}$ and $\mathcal{C}_{*}$.

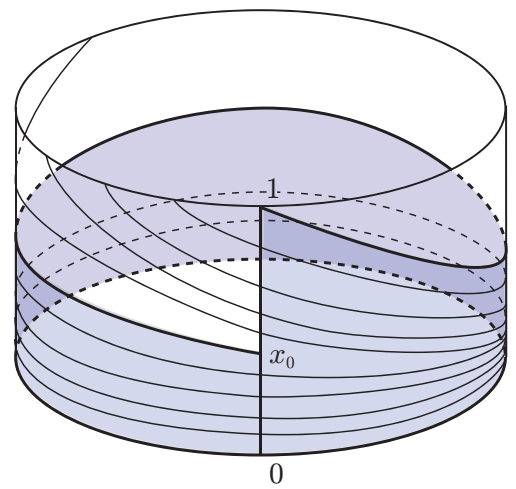

Figure 5. "Suspension" of a contraction. 
REMARK 4.10. Observe that any example of compact griddled 3-manifolds we have obtained so far is one of the following list:

i) $\mathbb{D}^{2} \times[0,1], \mathbb{D}^{2} \times \mathbb{S}^{1}, \mathbb{S}^{2} \times[0,1]$ or $\mathbb{T}^{2} \times[0,1]$ if $\partial M \neq \emptyset$,

ii) $\mathbb{S}^{2} \times \mathbb{S}^{1}$ or any $\mathbb{S}^{1}$-bundle over $\mathbb{T}^{2}$ if $\partial M=\emptyset$.

Moreover on $\mathbb{D}^{2} \times[0,1]$ or $\mathbb{S}^{2} \times[0,1]$ there is just one griddled structure while on all others, there exist countably many different ones.

We come to our classification result. Take again a connected component $\Sigma_{0}$ of the singular set $\Sigma$ of the griddled foliated manifold $(M, \mathcal{F}, \mathcal{C})$ and observe that the $\mathcal{F}$ saturation $\Omega_{0}$ of $\Sigma_{0}$ is an open $\mathcal{F}$-saturated subset of $(M, \mathcal{F})$ thus its closure $\bar{\Omega}_{0}$ is saturated as well and we have two partial results:

LEMMA 4.11. If some leaf $L \subset \Omega_{0}$ is a disk or a sphere, then $\bar{\Omega}_{0}=M$ and $(M, \mathcal{F})$ is the trivial $L$-bundle over $\mathbb{S}^{1}$ or $[0,1]$ with its canonical griddled structure.

Proof. The first part of the statement is a direct consequence of the global Reeb stability theorem in codimension 1 (see for example $[\mathbf{C C}]$ ). We identify the griddled structure using Lemmas 4.4 and 4.8.

LEMMA 4.12. If some leaf $L \subset \Omega_{0}$ is a plane, then $\bar{\Omega}_{0}$ is a canonically griddled Reeb component.

Proof. Of course any leaf in $\Omega_{0}$ admits a singularity and having one planar leaf, we deduce from Lemma 4.11, that all leaves in $\Omega_{0}$ are planes. Moreover because the griddled structure of a plane has just one singularity, these planes cut $\Sigma_{0}$ in exactly one point, so they are proper leaves closed in $\Omega_{0}$. Consequently as $M$ is compact, $L$ cannot be closed in $M$ and therefore $\Omega_{0} \neq M$. Now, there exists a transverse arc $\gamma:[0,1] \rightarrow M$ which does not meet $\Sigma$ and verifies

$$
\left.\left.\gamma(0) \in \bar{\Omega}_{0} \backslash \Omega_{0} \quad \text { and } \quad \gamma(t) \in \Omega_{0} \quad \text { for } \quad t \in\right] 0,1\right] .
$$

We make two observations:

(1) According to Lemma 4.11, the leaf $L_{\gamma(0)}$ through $\gamma(0)$ is neither a disk nor a sphere. Nor is it a plane because if this would be the case, $L_{\gamma(0)}$ would meet a component $\Sigma_{1}$ of $\Sigma$ which by continuity would also meet the nearby planes $L_{\gamma(t)}$ for small $t$. But any such plane meets $\Sigma$ in a unique point, so we would conclude that $\Sigma_{1}=\Sigma_{0}$ contradicting the fact that $L_{\gamma(0)}$ is not contained in $\Omega_{0}$.

(2) From (1) above, we deduce that $L_{\gamma(0)}$ is a torus or a cylinder and the leaf $c_{0} \in \mathcal{C}$ through $\gamma(0)$ is not null homotopic in $L_{\gamma(0)}$. But according to Lemma 4.7, its holonomy transformation is trivial so that the one parameter family $\Gamma=\left\{c_{\gamma(t)}\right\}_{t \in[0,1]}$ is a continuous family of embedded circles such that $c_{\gamma(t)}$ is null-homotopic in $L_{\gamma(t)}$ because the latter is a plane for any $t \in] 0,1]$. In other words, the loop $c_{\gamma(0)}$ is a non trivial vanishing cycle which by Novikov's celebrated theorem, is supported by a 2-torus bounding a Reeb component (note that Novikov's theorem initially stated for $C^{2}$-foliations (see $[\mathbf{N}]$ ), remains valid in class $C^{0}$ see $\left.[\mathbf{A H S}]\right)$. The final result follows using Lemma 4.9. 
LEMMA 4.13. If $\mathcal{F}$ has at least one planar leaf, $M$ will be obtained by gluing one or two griddled Reeb components along the boundaries of some regularly griddled $\mathbb{T}^{2} \times[0,1]$, possibly reduced to a single torus. Moreover $M$ is diffeomorphic to $\mathbb{D}^{2} \times \mathbb{S}^{1}$ or $\mathbb{S}^{2} \times \mathbb{S}^{1}$ with one of the structures described in (3) of Construction 3.4.

Proof. According to Lemma 4.11, any leaf of $\mathcal{F}$ is a torus, a cylinder or a plane and by Lemma 4.12, any plane is contained in a griddled Reeb component. Now as $M$ is compact, it contains at most finitely many Reeb components and if we cut them off, the remainder will be a compact saturated submanifold $N$ supporting a regularly griddled foliation. If $N$ is empty, $M$ will be obtained by gluing two Reeb components, on the other hand, if $N \neq \emptyset$, Lemma 4.2 implies that any connected component $N_{0}$ of $N$ is an orientable $\mathbb{S}^{1}$-bundle over $\mathbb{A}$, thus $N_{0} \cong \mathbb{T}^{2} \times[0,1]$ and $\partial N_{0}$ is the union of two griddled tori. If we stick $N_{0}$ together with at most two Reeb components, we obtain a closed manifold. We conclude that $N$ is indeed connected, diffeomorphic to $\mathbb{T}^{2} \times[0,1]$ and $M$ is obtained by gluing one or two griddled Reeb components along the boundary components of $N$ : the resulting manifold is $\mathbb{D}^{2} \times \mathbb{S}^{1}$ or $\mathbb{S}^{2} \times \mathbb{S}^{1}$ with the structure described in (3) of Construction 3.4.

We now reach to the wanted classification (compare with Theorem A):

THEOREM 4.14. The list of griddled foliated 3-manifolds described in Section 3 is complete. More precisely, a compact 3-manifold $M$ supports a griddled foliation of class $C^{1}$ if and only if $M$ is diffeomorphic to one of the following manifolds:

i) $\mathbb{D}^{2} \times[0,1], \mathbb{D}^{2} \times \mathbb{S}^{1}, \mathbb{S}^{2} \times[0,1]$ or $\mathbb{T}^{2} \times[0,1]$ if $\partial M \neq \emptyset$,

ii) $\mathbb{S}^{2} \times \mathbb{S}^{1}, \mathbb{T}^{3}$ or any $\mathbb{S}^{1}$-bundle over $\mathbb{T}^{2}$ if $\partial M=\emptyset$.

Moreover if $M$ is either $\mathbb{D}^{2} \times[0,1]$ or $\mathbb{S}^{2} \times[0,1]$, all griddled structures on $M$ are $C^{0}$ isotopic while in the remaining cases, $M$ supports countably many different $C^{0}$-isotopy and $C^{0}$-conjugacy classes of griddled structures.

Proof. If $\mathcal{F}$ has a leaf homeomorphic to $\mathbb{D}^{2}$ or $\mathbb{S}^{2}$, we conclude by Lemma 4.11 and if $\Sigma$ is empty we apply Lemma 4.2. So, according to Lemma 4.12, it remains to consider the case when $\mathcal{F}$ has a planar leaf and we conclude using Lemma 4.13.

Concerning the number of isotopy classes of griddled structures, we remark that the only allowed codimension 1 foliations on $\mathbb{D}^{2} \times[0,1]$ or $\mathbb{S}^{2} \times[0,1]$ are just the trivial fibrations: this implies that any griddled structure is a canonical one (see Remark 4.10), on the other hand all other manifolds which are considered contain eventually a submanifold diffeomorphic to $\mathbb{T}^{2} \times[0,1]$, thus support infinitely many griddled structures according to Remark 4.10.

It is worth noting that the 3 -sphere does not support any griddled structure.

\section{Topological canal foliations on 3-manifolds.}

Consider a finite family $\mathcal{D}=\left\{F_{1}, F_{2}, \ldots, F_{k}\right\}$ of compact leaves of a codimension 1 compact foliated manifold $(M, \mathcal{F})$. Cutting $M$ along these leaves, we produce a fi- 
nite family $\left\{N_{1}, N_{2}, \ldots, N_{l}\right\}$ of compact foliated manifolds and a foliation preserving submersive map

$$
\psi: \coprod_{j} N_{j} \rightarrow M
$$

The restriction of $\psi$ either to the interior or to any boundary component of any $N_{j}$ is injective. We call $\psi$ a foliated decomposition of $(M, \mathcal{F})$ defined by the fundamental family $\mathcal{D}$ of compact leaves. Now we introduce our last concept to be considered:

Definition 5.1. A codimension 1 foliation $\mathcal{F}$ on a compact connected 3-manifold $M$ (possibly with boundary) is a topological canal foliation if there exists a foliated decomposition $\psi: \coprod_{j} N_{j} \rightarrow M$ of $(M, \mathcal{F})$ defined by a fundamental family $\mathcal{D}$ of compact leaves verifying the two following conditions:

i) for each $j$, the foliation $\mathcal{F}_{j}$ induced by $\mathcal{F}$ on $N_{j}$ is tangent to the boundary, admits a griddled structure $\mathcal{C}_{j}$ and any component of $\partial N_{j}$ is a regularly griddled torus,

ii) any fundamental torus $F_{i} \in \mathcal{D}$ being the image by $\psi$ of two boundary components of $\coprod_{j} N_{j}$ will be endowed with two griddled structures and these structures are transverse to each other.

The elements of $\mathcal{D}$ are called the turning leaves of $\mathcal{F}$ and the manifolds $N_{j}$ are its griddled components.

One justification for this definition is that we want geometric canal foliations of the 3 -sphere to be a special case of topological canal foliations. Note also that our topological canal foliations may be of any differentiability class.

ExAmple 5.2. (1) Taking the empty fundamental family of compact leaves, we see immediately that any griddled foliation is a topological canal foliation.

(2) Any geometric canal foliation of $\mathbb{S}^{3}$ where no planar leaf of a Reeb component has a spherical cap, that is a disc isometric to a piece of a round sphere, is a topological canal foliation (see Theorems 4.2.1 and 4.2.3 in [LW2]). Indeed, in a geometric canal foliation, there exists a finite number of Dupin cyclides $D_{i}$ with two orthogonal griddled structures induced and the pieces obtained by cutting along these Dupin cyclides are griddled.

(3) Next we adapt Construction 3.4 to this more general setting: we glue together griddled pieces along tangential boundary components with the weaker requirement that the gluing maps either identify the corresponding griddled structures or map one transversely to the other. The manifold obtained by this procedure will be endowed with a topological canal foliation.

There are two particularly interesting cases:

(3.1) as any lens space is obtained by sticking together two solid tori, thus we can define a canal foliation on any lens space by gluing conveniently two griddled Reeb components (with flat holonomy); its singular set will be a link with two branches; 
(3.2) identifying the two boundary components of some griddled $\mathbb{T}^{2} \times[0,1]$, we construct similarly a regular canal foliation on any $\mathbb{T}^{2}$-bundle over $\mathbb{S}^{1}$.

Putting together the classification Theorem 4.14 of griddled 3-manifolds and the results of construction (3) in the above Example 5.2, we will obtain a complete description of all manifolds which support topological canal foliations. Recall that a canal foliation is regular if it is without singularities, then the following is an analogue of Lemma 4.13.

LEMMA 5.3. Let $(M, \mathcal{F})$ be a topological canal foliation on a compact manifold $M$. If $\mathcal{F}$ has a planar leaf, $M$ will be obtained by gluing one or two griddled Reeb components along the boundaries of some $\mathbb{T}^{2} \times[0,1]$, possibly reduced to a single leaf, and endowed with a regular topological canal foliation tangent to the boundary. Moreover $M$ is diffeomorphic to $\mathbb{D}^{2} \times \mathbb{S}^{1}, \mathbb{S}^{2} \times \mathbb{S}^{1}, \mathbb{S}^{3}$ or any lens space.

Proof. Observe that any griddled component of $\mathcal{F}$ is $\mathcal{F}$-saturated, any planar leaf of $\mathcal{F}$ is contained in a griddled Reeb component and Lemmas 4.11, 4.12 and 4.13 can be adapted to topological canal foliations. Thus proceeding exactly as in the proof of Lemma 4.13, we conclude that $M$ is obtained by sticking together one or two Reeb components with a manifold $P=\mathbb{T}^{2} \times[0,1]$ endowed with a topological canal foliation without singularities. The class of resulting manifolds is larger than that of griddled foliated manifolds (see Lemma 4.13) because of the weaker requirements on the gluing maps; in particular we obtain all lens spaces as observed in (3.1) of Example 5.2.

Again, we get the following complete classification (compare with Theorem B):

THEOREM 5.4. A topological canal foliation of class $C^{1}$ on a compact manifold is either griddled or obtained by gluing two or three compact griddled foliated pieces. Therefore a compact 3-manifold $M$ supports such a foliation if and only if it is diffeomorphic to one of the following:

i) $\mathbb{D}^{2} \times[0,1], \mathbb{D}^{2} \times \mathbb{S}^{1}, \mathbb{S}^{2} \times[0,1]$ or $\mathbb{T}^{2} \times[0,1]$ if $\partial M \neq \emptyset$,

ii) $\mathbb{S}^{2} \times \mathbb{S}^{1}, \mathbb{S}^{3}$ or any lens space, $\mathbb{T}^{3}$ or any $\mathbb{S}^{1}$-bundle over $\mathbb{T}^{2}$ if $\partial M=\emptyset$.

Moreover all manifolds $M$ listed in ii) support countably many different $C^{0}$-isotopy and $C^{0}$-conjugacy classes of such foliations.

OBSERVATION 5.5. The meaning of $\cong$ depends on the differentiability class of a foliation under consideration. In Theorem 5.4, the foliation is of class $C^{1}$, we make the same assumption in this observation, so $\cong$ should mean "diffeomorphic of class $C^{1}$ ".

(1) Observe that any topological canal foliation $(M, \mathcal{F})$ on a closed manifold $M$, is of one of the following two types:

a) either it admits a maximal regular component $P \cong \mathbb{T}^{2} \times[0,1]$, possibly reduced to a single toral leaf,

b) or it is a $\mathbb{T}^{2}$-bundle over $\mathbb{S}^{1}$ thus is the quotient of a maximal regular component $P \cong \mathbb{T}^{2} \times[0,1]$ by a monodromy map $\varphi: \mathbb{T}^{2} \times\{0\} \rightarrow \mathbb{T}^{2} \times\{1\}$. 
In both cases, we call $P$ the kernel of $(M, \mathcal{F})$.

(2) Now write $\mathbb{T}^{2}$ as a product $A \times B$ of two circles and lift this product structure to all toral leaves in the kernel $P \cong \mathbb{T}^{2} \times[0,1]$; we will say that it defines a coherent system of meridians and parallels on $P$.

Using the previous notion, we introduce a particular class of topological canal foliations:

Definition 5.6. i) A griddled structure on $\mathbb{T}^{2} \times[0,1]$ endowed with a coherent system of meridians and parallels will be called of meridian (resp. parallel) type if all its fibers are homotopic to $A$ (resp. to $B$ ).

ii) A topological canal foliation $\mathcal{F}$ on $M$ will be called of strong type if there exists a coherent systems of meridians and parallels on the kernel of $\mathcal{F}$ such that the griddled structure induced on any toral leaf of $\mathcal{F}$ is either of meridian or of parallel type.

Now, observe that the group $\Gamma$ of isotopy classes of orientation preserving diffeomorphisms of $\mathbb{T}^{2}$ which preserve a product structure $\mathbb{T}^{2}=A \times B$ identifies with the group of matrices

$$
\left\{ \pm\left(\begin{array}{cc}
0 & 1 \\
-1 & 0
\end{array}\right), \pm I d\right\} .
$$

Thus, for canal foliations of strong type, Theorem 5.4 reduces to the following:

COROLlary 5.7. There are only finitely many closed 3-manifolds $M$ which support a topological canal foliation of strong type; precisely $M$ will be diffeomorphic to one of the following:

i) $\mathbb{S}^{3}$ or $\mathbb{S}^{2} \times \mathbb{S}^{1}$,

ii) a $\mathbb{T}^{2}$-bundle over $\mathbb{S}^{1}$ with monodromy map $\varphi$ whose isotopy class belongs to $\Gamma$.

But on each of these manifolds there are infinitely many different $C^{0}$-isotopy or $C^{0}$-conjugacy classes of canal foliations of strong type.

Recall that geometric canal foliations were supposed to be of class $C^{2}$, and we get Theorem C:

TheOREM 5.8. A topological canal foliation $\mathcal{F}$ of class $C^{2}$ on $\mathbb{S}^{3}$ is $C^{0}$-conjugate to a geometric one if and only if it is of strong type.

Proof. The characteristic circles of a geometric canal foliation on $\mathbb{S}^{3}$ define a coherent system of meridians and parallels on its kernel and thus any geometric canal foliation is a topological canal foliation of strong type (see [LW2]).

To prove the converse statement, recall that a topological canal foliation $\mathcal{F}$ of strong type on $\mathbb{S}^{3}$ will be obtained by gluing two griddled Reeb components along the boundary components of its kernel $P \cong \mathbb{T}^{2} \times[0,1]$. As $\mathcal{F}$ is of strong type, $P$ will admit a coherent system of meridians and parallels and the two Reeb components will be one of meridian and the other of parallel type. 
Then the conformal structure on the torus defined by the product decomposition $\mathbb{T}^{2}=A \times B$ extends to all leaves of $\mathcal{F}$ and defines a structure of geometric canal foliation on $\mathcal{F}$.

Finally we construct a smooth topological canal foliation on $\mathbb{S}^{3}$ which is not conjugate to a geometric one.

Example 5.9. Write $\mathbb{T}^{2}=A \times B$ as in Definition 5.6 and consider the trivial fibration $\mathcal{C}_{*}$ by circles $\pi: P=\mathbb{T}^{2} \times[0,1] \rightarrow \mathbb{A}$ whose fibers are in the homotopy class of $A+B$. According to example (1) in Example 3.3, there exists a codimension 1 foliation $\mathcal{F}_{*}$ on $P$ tangent to the boundary such that $\mathcal{C}_{*}$ is a griddled structure on $\mathcal{F}_{*}$. We glue a griddled Reeb component $\mathcal{R}_{0}$ along $\mathbb{T}^{2} \times\{0\}$ in such a way that the griddled structure on $\partial \mathcal{R}_{0}$ is identified with a fibration of meridian type of $\mathbb{T}^{2} \times\{0\}$ and a second one $\mathcal{R}_{1}$ along $\mathbb{T}^{2} \times\{1\}$ with the griddled structure of $\partial \mathcal{R}_{1}$ identified with a parallel fibration. We obtain a foliated closed 3 -manifold $(M, \mathcal{F})$ which is the 3 -sphere endowed with a topological canal foliation $\mathcal{F}$. It is not a geometric one because it is not of strong type.

As no manifold listed in Theorem 5.8 admits a hyperbolic metric, we get:

COROLlaRY 5.10. There are no canal foliations on compact hyperbolic 3-manifolds.

\section{The non-orientable case.}

There are three ways to enlarge the family of griddled or canal foliated manifolds by allowing the griddled structure $\mathcal{C}$, the codimension 1 foliation $\mathcal{F}$ or even the manifold $M$ to be non orientable. We describe next some new examples and new phenomena which will occur already when keeping $M$ orientable.

ExAmple 6.1. (1) Let $\sigma: \mathbb{S}^{2} \rightarrow \mathbb{S}^{2}$ be the diffeomorphism of the unit sphere $\mathbb{S}^{2} \subset \mathbb{R}^{3}(x, y, z)$ obtained by composing the symmetries with respect to the planes $y=0$ and $z=0$. It enjoys the following properties:

a) $\sigma$ is orientation preserving thus isotopic to the identity,

b) it preserves globally the canonical griddled structure $\mathcal{C}_{*}$ of $\mathbb{S}^{2}$ but reverses its orientation.

Thus the $\mathbb{S}^{2}$-bundle $\mathcal{F}_{0}$ over $\mathbb{S}^{1}$ with monodromy $\sigma$ is the trivial bundle endowed with a non orientable griddled structure $\mathcal{C}_{0}$ and as $\sigma$ exchanges the two singularities of $\mathcal{C}_{*}$, the singular set $\Sigma$ of $\mathcal{C}_{0}$ is connected.

As in the orientable case, the singular set $\Sigma$ admits a neat tubular neighborhood $\tau(\Sigma)$ and the compact griddled manifold $N=M \backslash \operatorname{int}[\tau(\Sigma)]$ will be an orientable bundle $\mathcal{F}_{1}$ over the circle with fiber the annulus $\mathbb{A}=\mathbb{S}^{1} \times[-1,+1]$. Its monodromy exchanges the two boundary components of $\mathbb{A}$ so that the boundary of $N$ is connected and is indeed a torus. This monodromy preserves globally the natural fibration of $\mathbb{A}$ by circles thus induces a canonical regular griddled structure $\mathcal{C}_{1}$ supported by $\mathcal{F}_{1}$.

(2) Take the griddled manifold $\left(N, \mathcal{F}_{1}, \mathcal{C}_{1}\right)$ described above; the foliation $\mathcal{F}_{1}$ is transverse to the boundary; we make it spiral along $\partial N$ and obtain a foliation $\mathcal{F}$ tangent to 
the boundary whose non compact leaves are cylinders: it is the cylindrical component of Moussu-Roussarie (see $[\mathbf{M R}]$ ). It is not difficult to see that we may assume the spiraling to be compatible with the fibration $\mathcal{C}_{1}$ and we obtain a regular griddled structure $\mathcal{C}$ supported by $\mathcal{F}$ which is tangent to the boundary of $N$; we call $(N, \mathcal{F}, \mathcal{C})$ a griddled cylindrical component.

More generally, the total space of a non-orientable $\mathbb{S}^{1}$-bundle over the Klein bottle or the Möbius band will be orientable thus providing more examples of regular griddled foliated manifolds.

(3) Gluing a $(N, \mathcal{F}, \mathcal{C})$ with a griddled Reeb component we obtain a non orientable griddled structure on $\mathbb{S}^{2} \times \mathbb{S}^{1}$ whose singular set is now connected.

\section{References}

[AHS] F. Alcalde, G. Hector and P. Schweitzer, Vanishing cycles and generalized Reeb components, preprint, 2017.

[BLW] A. Bartoszek, R. Langevin and P. Walczak, Special canal surfaces of $S^{3}$, Bull. Braz. Math. Soc., 42 (2011) 301-320.

[BW] A. Bartoszek and P. Walczak, Foliations by surfaces of a peculiar class, Ann. Polon. Math., 94 (2008), 89-95.

[CSW] G. Cairns, R. W. Sharpe and L. Webb, Conformal invariants for curves in three dimensional space forms, Rocky Mountain J. Math., 24 (1994), 933-959.

[CC] A. Candel and L. Conlon, Foliations I, II, Amer. Math. Soc., Providence, 2000 and 2003.

[EMS] R. D. Edwards, K. C. Millet and D. Sullivan, Foliations with all leaves compact, Topology, 16 (1977), 13-32.

[E] D. B. A. Epstein, Periodic flows on three-manifolds, Annals of Math., 95 (1972), 66-82.

[H] G. Hector, Feuilletages en cylindres, In: Geometry and Topology, Rio de Janeiro, 1976, Lecture Notes in Math., 597, Springer, 1977, 252-270.

[HC] G. Hector and M. A. Chaouch, Dynamiques source-puits et flots transversalement affines, Contemp. Math., 498 (2009), 99-126.

$[\mathrm{HH}]$ G. Hector and U. Hirsch, Introduction to the geometry of foliations, Part A and B, Vieweg, 1981.

[Kn1] H. Kneser, Reguläre Kurvenscharen auf die Ringflachen, Math. Annalen, 91 (1923), $135-154$.

[Kn2] H. Kneser, Die Deformationssätze der einfach zuzammenhängenden Flächer, Mat. Z., 23 (1926), $362-372$.

[Kr] R. Krasauskas, Minimal rational parametrizations of canal surfaces, Computing, 79 (2007), 281-290.

[LW1] R. Langevin and P. Walczak, Conformal geometry of foliations, Geom. Dedicata, 132 (2008), 135-178.

[LW2] R. Langevin and P. Walczak, Canal foliations of $\mathbb{S}^{3}$, J. Math. Soc. Japan, 64 (2012), 659-682.

[MR] R. Moussu and R. Roussarie, Relations de conjugaison et de cobordisme entre certains feuilletages, Inst. Hautes Études Sci. Publ. Math., 43 (1974), 143-168.

[N] S. P. Novikov, Topology of foliations, Trans. Moscow Math. Soc., 14 (1965), 268-304.

[PP] M. Peternell and H. Pottmann, Computing rational parametrization of canal surfaces, J. Symb. Comp., 23 (1997), 255-266.

[Ro] D. Rolfsen, Knots and links, Publish or Perish, Berkeley, 1975.

[Sm] S. Smale, Diffeomorphisms of the 2-sphere, Proc. Amer. Math. Soc., 10 (1959), 621-626.

[Ze1] A. Zeghib, Laminations et hypersurfaces géodésiques des variétés hyperboliques, Ann. Sci. École Norm. Sup., 24 (1991), 171-188.

[Ze2] A. Zeghib, Sur les feuilletages géodésiques continus des variétés hyperboliques, Invent. Math., 114 (1993), 193-206. 


\section{Gilbert Hector}

Institut C. Jordan

UMR CNRS 5208 Université Lyon 1

Lyon, France

E-mail: gilberthector@orange.fr

\section{Rémi LANGEVIN}

Institut de Mathématiques de Bourgogne UMR CNRS 5584 Université de

Bourgogne Franche Comté

Dijon, France

E-mail: Remi.Langevin@u-bourgogne.fr

\section{Paweł WALCZAK}

Katedra Geometrii, Wydział Matematyki i Informatyki

Uniwersytet Łódzki

Łódź, Poland

E-mail: pawel.walczak@wmii.uni.lodz.pl 